\title{
Morphometric studies with pure bred stock of Apis mellifera carnica Pollmann from Hessen
}

\author{
V Maul, A Hähnle \\ Hessische Landesanstalt für Tierzucht, Abteilung für Bienenzucht, Erlenstraße 9. \\ 35274 Kirchhain, Germany
}

\begin{abstract}
Summary - Breeder colonies were studied to determine whether pure Carnica, according to the breeding guidelines, might be hybrids between Carnica and Mellifera according to other characteristics not considered by the guidelines. For this purpose, a total of 123 samples of 30 bees each from Hessian Carnica breeder colonies were checked morphometrically according to the guidelines 10 the Deutscher Imkerbund. In addition, 10 other characteristics of the wing veination which are not under control of the breeders were determined and compared to typical Apis mellifera carnica and A $\mathrm{m}$ mellifera from the biometric data bank of Oberursel. Results: 1) Being of high interest to the breeders, and as a consequence of selection, the characteristic 'cubital index' shows considerably higher values than in A m carnica; 2) from discriminant analyses considering other characteristics than the cubital index, a high degree of similarity appears between the samples from Hessen and typical $A$ m carnica. Deviations from $A m$ carnica tend rather to other directions than toward $A m$ mellifera; 3 ) as judged by frequency distributions of single characteristics, however, similarities with $A m$ mellifera can also be seen. A hybridisation with $A m$ mellifera cannot be safely excluded, therefore, but seems to play a minor role only. An influence of genes from other races than Mellifera is more likely.
\end{abstract}

Apis mellifera carnica / Apis mellifera mellifera / morphometry / pure race breeding / mating control

\section{INTRODUCTION}

The consideration of exterior characteristics has a long tradition in German bee breeding. Starting from the differentiation of geographical races, exterior standards for single stock lines were being tried to establish, however without success (Goetze, 1940; Reichsfachgruppe Imker EV, 1935). After 1945, the interest was limited again to the differentiation of races. Pure race breeding of Apis mellifera carnica from the Eastern Alpine region was more and more favoured instead of the autochthonous $A \mathrm{~m}$ mellifera (Dreher, 1946). The determination of certain external characteristics and their evaluation according to a fixed standard should serve mainly as a means of mating control: deviations from the characteristic pattern in the worker offspring of a given queen mated on a land mating station should be interpreted as mismatings, and the respective queens should be excluded from the stock (Deutscher Imkerbund, 1954). The further development of the breeding guideline's content followed the progress of 
knowledge (Dreher, 1950; Ruttner, 1963, 1969; Deutscher Imkerbund, 1971, 1988). From the practical application of the guidelines by active breeders, landwide a strong effect of replacement resulted against the original land bee ( $A \mathrm{~m}$ mellifera or its uncontrolled descendants, respectively). This occurred even in populations which were not directly controlled by breeders (Reinsch et al, 1991).

Based on a study of bee populations from Lower Bavaria, Moritz (1991) has drawn attention to the limitations of the concept of using land mating stations in combination with morphometry for the purpose of pure race breeding. He concludes that the populations tested by himself inside and marginally of pure race areas appear as typical Carnica, according to the characteristics controlled by the breeders. Using other characteristics of the wing veination, however, they should be considered as hybrids between Carnica and Mellifera. Due to the basic importance for practical bee breeding in Germany, this complementary study was performed with pure Carnica stock from Hessen, where mating control occurs partly through land mating stations, partly through instrumental insemination. A direct comparison should be made with the pattern of characteristics of autochthonous Carnica and Mellifera populations, based on the morphometric data bank of Oberursel (Ruttner, 1988a).

\section{MATERIALS AND METHODS}

\section{Samples}

Samples of 30 newly emerged worker bees were collected from 123 colonies out of 18 pure breeding apiaries in Hessen during August and September 1992. The bees were kept at room temperature in darkened cages until hardening of the chitinous parts, then freeze killed and stored at $-12^{\circ} \mathrm{C}$ under protection against evaporation. Four of the 18 breeding apiaries ( 45 samples) maintain their stock predominantly or exclusively by means of artificial insemination. The other 14 (78 samples) use land mating stations. Thus, two subgroups were formed for the later calculations, Hessen $(B)=$ land mating station and Hessen (I) $=$ instrumental insemination. Only these colonies were used for sampling which were either approved as breeder colonies or selected by the breeder for the purpose of approval.

Reference samples for the presented study were taken from the morphometric data bank of Oberursel, 23 Carnica samples from the Alpine region and 15 Mellifera samples from Norway.

\section{Data collection}

First, all Hessian samples were tested according to the breeding guidelines of the Deutscher Imkerbund (1988) according to Ruttner (1983, 1988b). This includes the following characteristics: cubital index, length of overhair on the 5th tergite, width of tomentum band on the 4th tergite, colour markings on the abdomen. Furthermore, the same characteristics of the wing veination were determined according to Dupraw (1965a, b), as they were registered in the data bank of Oberursel. These were the following angles: A4, B4, D7, E9, G18, K19, J10, J16, L13, N32, O26.

The measurements were performed with the specifically installed computer system at the Institut für Bienenkunde Oberursel (Meixner, 1994). The left forewings were mounted on glass slides and shown on a monitor via a CCD-camera. The wanted intersection points were marked by means of the mouse in a fixed sequence, so that the program could calculate and store the data of the single angles. Only the means of each sample were analysed with a discriminant analysis from the SPSS-PC, $\vee 4.01$ package. Information on the frequency distribution, eg asymmetry or side peaks, was not considered in the discriminant analysis. It was tried, however, to consider single samples on the level of the single data and to compare with the respective total from the data bank. For this purpose, classes of $1^{\circ}$ width were formed for each angle and the frequency distributions determined. This procedure is analogous to the common procedure in evaluating the cubital index of single samples for the purpose of selection. 


\section{RESULTS}

According to the breeding guidelines (Deutscher Imkerbund, 1988), all samples appeared as 'corresponding to the race standard'. It is conspicuous that the frequency distributions of the cubital index (Cl) often are considerably beyond the limits set by the standard (thereafter, only $15 \%$ of all cases should occur in classes 15-17, only less than $2 \%$ should be in classes below 15 , and the average should be larger than 2.5). Figure 1 comprises all data into a relative frequency distribution and shows the shifting compared to the Carnica samples from the data bank. The subgroups Hessen (B) and Hessen (I) cannot be distinguished in the distribution of this characteristic. Details of the other characteristics are not presented here.

Discriminant analyses with all tested characteristics show a very strong influence of the $\mathrm{Cl}$. Since the $\mathrm{Cl}$ is obviously influenced by selection (compare Bienefeld, 1992), it is no longer regarded in the further calculations. Also the angles N23 and J16 were disregarded in further calculations, since an influence of the investigator on the fixation of the common intersection point could not be excluded.

Figure 2 shows the result of the discriminant analysis with the remaining characteristics for the 4 groups A m mellifera, A m carnica, Hessen (B) and Hessen (I). The three Carnica groups are very similar and all well separated from the Mellifera group. There is a tendency, however, that the two groups from Hessen are separated from the reference Carnica in opposite directions along the axis of function 2. Table I shows the result of classification of the discriminant analysis for the four groups. Whereas all Mellifera samples are clearly separated, overlappings occur between the Carnica groups which are similar to each other. Table II shows for all four groups the means and standard deviations of all the characters tested.
Analogously to the procedure of the breeding guidelines and as a model only, all characteristics of some samples were considered on the level of single data. Samples No 22 (mating station) from the center of the 'Hessen cluster' and No 111 (mating station) from the margin toward Mellifera were selected as examples. Figures $3 a, b$ show the frequency distributions per class of the angle A4 compared to the respective distributions of the totals of Mellifera, Carnica and Hessen. Sample 22 appears to be very similar to Carnica and Hessen, both clearly different from Mellifera (fig 3a). Sample 111, on the contrary, is more similar to the Mellifera distribution (fig 3b). Similar situations can be found with single Carnica samples from the data bank which are positioned marginally in the Camica cluster toward Mellifera.

\section{DISCUSSION}

According to the presented discriminant analyses, the samples from Hessian pure bred stocks are not positioned between Carnica and Mellifera, but very closely to Carnica. A hybridisation between Carnica and Mellifera cannot be derived from the data.

Hints of a hybridisation could eventually be deduced from a comparison between frequency distributions of single characteristics and the respective totals as a standard - analogously to the procedure of the breeding guidelines with the $\mathrm{Cl}$. Sample 111 could thus perhaps be considered as a Carnica-Mellifera-hybrid. This procedure requires still a verification by the analysis of special crossing experiments. It can be concluded that, if at all, only a very little part of Mellifera genome is present in the Hessian breeding population.

It is important for further considerations that the samples from Hessen are not positioned in full congruence with the typical Carnica and, furthermore, that the insemi- 
nated group tends into another direction than the group from land mating stations. Relatively to Mellifera and Carnica, Hessen (B) tends to a position which is similar to that of the Lower Saxonian land bee as described by Reinsch et al(1991), although on the basis of other characteristics. The authors point out that the genome of the land bee is not only influenced by Mellifera and Carnica, but also by other races or origins (eg Caucasica, Buckfast). Thus, it can be assumed that from this side non-Carnica genes have entered the breeding population. However, this still needs to be proved.

It can be excluded that the tendency of an opposite arrangement of the inseminated group Hessen (I) results from crosses with other races. As compared to the reference Carnica, the stock of Hessische Landesanstalt für Tierzucht (Kirchhain) tends to more negative values of function 1 (in the graph downward) and to more positive values of function 2 (in the graph right). Two groups derived from the Kirchhain stock and bred independently and rather closely form almost separate 'subclusters' at high positive values of function 2 (see special markings in fig 2). This distribution indicates more likely a random effect in the sense of genetic drift.

Many of the Hessian stocks from mating stations have been temporarily combined with Kirchhain stock for the purpose of regeneration. Taking the (not shown) cluster 'Kirchhain' as a reference instead of Carnica, the distance to Hessen (B) would be even larger. The assumption of an influence of another (non-Carnica, non-Mellifera) genome would have even more weight. However, effects of genetic drift cannot be fully excluded.

With regard to the initial question, it is important that Mellifera as the former main element of the land bee is no longer evident in the Carnica breeding population in a degree worth mentioning. Different from Lower Bavaria, the work with land mating stations combined with morphometric con- trols appears in Hessen as predominantly successful. It cannot be quantified to what extent propagations from inseminated stocks have contributed to this result in the course of more than 20 years. The comparison of the stocks from land mating stations with the inseminated ones suggests, however, that despite the correct characteristics according to the guidelines, some foreign genes have entered the land mating stocks.

Differences in the results, compared to Moritz (1991), are partly explained by the different materials studied: samples from Lower Bavarian protective belts on one side and samples from pure breeding stocks, preselected according to the guidelines on the other. To some extent, however, the differences can be influenced by the different choice of the reference samples. Moritz (1991) had used as six Carnica samples from the stock of Hessische Landesanstalt für Tierzucht (Kirchhain), arguing that this stock has always been maintained by insemination and should include most of the commercially used lines in Germany. The latter is certainly not valid. Above all, however, the present study has shown that the Kirchhain stock is more different from $\mathrm{Mel}$ lifera than the reference Carnica of the data bank. In case of including the cubital index into the calculations, the distance would be even larger.

For Mellifera, Moritz (1991) used seven museum samples collected in 1911/1912 at Erlangen and Kulmbach. They were chosen as representatives of the former land bee against which the breeding activities were directed in the sense of replacement grading. Proof is lacking, however, that they were truely original Mellifera without any influence of imported stock. For the presented study, therefore, the group 'Norway' from the data bank was chosen as representative of $A m$ mellifera. According to Rutther et al (1990), A $m$ mellifera has maintained a very uniform morphological pattern during its postglacial expansion, among oth- 
ers shown by comparison with samples from archeological excavations. The samples from Erlangen could not be included as further reference in the present study.

\section{CONCLUSIONS}

The standard values of the breeding guidelines are mainly designed to separate $\mathrm{Mel}$ lifera from Carnica. They are still bound to the data taken by Ruttner (1969) from autochthonous Carnica. To be valid further on in the sense of a control measure for land mating stations, they should better be bound to the actual characteristics of the present breeding population, particularly with regard to the increased cubital index. As a substitute, other characters should be included in the control. With regard to the necessary increase in labor, however, the breeders must consider whether this can be rational, since a permanent and absolutely safe control is not possible in this way, and reliable alternatives for the maintainance of the essential stocks do exist with island mating stations and insemination. This conclusion is congruent in tendency with the respective one of Moritz (1991), although the results of both studies are considerably different.

\section{ACKNOWLEDGMENTS}

The study was part of a diploma thesis of Andreas Hähnle guided by Prof Dr N Koeniger, Oberursel. We thank Mrs Marina Meixner for kindly offering the computer equipment and the data from the data bank of Oberursel, and $\mathrm{Mr}$ Nicolai Petersen as a beekeeping advisor for his support in collecting the samples. We also thank Prof Dr N Koeniger, Oberursel and Dr R Büchler, Kirchhain, for critically reading the manuscript

\title{
Deutsche Version
}

\section{Morphometrische Untersuchungen an Hessischen Reinzuchtbeständen von Apis mellifera carnica Pollmann}

\begin{abstract}
Zusammenfassung - Es sollte untersucht werden, ob bei Carnica-Reinzuchtvölkern trotz einwandfreier Befunde nach den Körrichtlinien eine verdeckte Hybridisierung mit Apis mellifera mellifera vorliegen kann. Dazu wurden an 123 Proben von je 30 Bienen aus hessischen Carnica-Reinzuchivölkern neben der Merkmalsprüfung gemäß den Zuchtrichtlinien des Deutschen Imkerbundes 10 in der Zuchtpraxis nicht beachtete Merkmale des Fiügelgeäders bestimmt und mit typischer $A \mathrm{~m}$ carnica und $A \mathrm{~m}$ mellifera aus der Datenbank Oberursel verglichen. Ergebnisse: 1) Das in der Zuchtpraxis stark beachtete Merkmal Cubitalindex ist selektionsbedingt gegenüber der ursprünglichen Carnica stark erhöht. 2) Aus Diskriminanzanalysen ohne Berücksichtigung des Cubitalindex ergibt sich eine sehr große Ähnlichkeit der Hessenproben mit $A$ m carnica, wobei die Abweichungen eher in andere Richtungen als zu A $m$ mellifera tendieren. 3) Im Bild der Häufigkeitsverteilung einzelner Merkmale ergeben sich allerdings bei einzelnen Proben Ähnlichkeiten mit $A m$ mellifera. Eine Hybridisierung mit $A m$ mellifera kann somit nicht ganz ausgeschlossen werden, dürtte aber nur eine unbedeutende Rolle spielen. Eher ist mit einem Einfließen von Genanteilen anderer Rassen zu rechnen.
\end{abstract}

Apis mellifera carnica / Apis mellifera mellifera / Morphometrie / Rassereinzucht / Paarungskontrolle 


\section{EINLEITUNG}

Die Berücksichtigung von äußeren Körpermerkmalen hat in der Bienenzüchtung Deutschlands eine lange Tradition. Ausgehend von der Unterscheidungsmöglichkeit geographischer Rassen versuchte man zunächst, auch für einzelne Zuchtstämme Exterieur-Standards festzulegen, blieb damit aber erfolglos (Goetze, 1940; Reichsfachgruppe Imker eV, 1935). Nach 1945 beschränkte man sich wieder auf die Unterscheidung nach der Rassenzugehörigkeit und favorisierte zunehmend die Reinzucht der aus dem Ostalpenraum stammenden Rasse Apis mellifera carnica anstelle der autochthonen Rasse Apis mellifera mellifera (Dreher, 1946). Die Untersuchung bestimmter Körpermerkmale und deren Bewertung nach einem festgelegten Standard sollte dabei vor allem als Mittel der Paarungskontrolle dienen: Abweichungen im Merkmalsbild der Arbeiternachkommen einer auf der Belegstelle begatteten Reinzuchtkönigin waren als Fehlpaarungen zu interpretieren und sollten zum Ausschluß der betroffenen Königinnen aus der Zuchtpopulation führen (Deutscher Imkerbund, 1954). Die inhaltliche Weiterentwicklung der Zuchtrichtlinien folgte später dem Fortschritt des Kenntnisstandes (Dreher, 1950; Ruttner, 1963, 1969; Deutscher Imkerbund, 1971, 1988). In der praktischen Umsetzung der Zuchtrichtlinien durch aktive Züchter ergab sich landesweit ein deutlicher Verdrängungseffekt gegen die ursprüngliche Landrasse ( $A \mathrm{~m}$ mellifera bzw ihre unkontrollierten Abkömmlinge), und dies auch in solchen Beständen, die nicht direkt von züchterischen Aktivitäten berührt waren (Reinsch et al, 1991).

Auf der Grundlage einer Untersuchung an niederbayerischen Bienenbeständen hat Moritz (1991) auf die Grenzen des nach den Zuchtrichtlinien verfolgten Konzepts der Rassereinzucht hingewiesen. Er kommt zu dem Schluß, daß die von inm untersuchten
Bestände im Innern und am Rande von Reinzuchtgebieten zwar nach den von den Züchtern überwachten Merkmalen als carnicatypisch einzustufen sind, nach anderen Merkmalen des Flügelgeäders aber als Hybriden zwischen Mellifera und Carnica anzusehen sind.

Wegen der grundsätzlichen Bedeutung für die praktische Bienenzüchtung in Deutschland wurde diese ergänzende Untersuchung an Reinzuchtbeständen aus Hessen durchgeführt, wo die Paarungskontrolle teils über Landbelegstellen, teils über instrumentelle Besamung erfolgt. Sie sollte einen direkten Vergleich mit dem Merkmalsbild autochthoner Carnica- und Mellifera-Bestände auf der Grundlage der Datenbank Oberursel ermöglichen (Ruttner, 1988a).

\section{MATERIAL UND METHODEN}

\section{Probenmaterial}

Im August und September 1992 wurden bei 18 hessischen Reinzuchtbetrieben aus insgesamt 123 Völkern Proben von je 30 frischgeschlüpften Arbeitsbienen entnommen. Die Bienen wurden bis zur Chitinaushärtung in abgedeckten Käfigen bei Zimmertemperatur gehalten, dann durch Einfrieren abgetötet und, vor Austrocknung geschützt, bei $-12^{\circ} \mathrm{C}$ gelagert. Von den 18 Zuchtbetrieben führen 4 (entsprechend 45 Proben) ihren Bestand überwiegend oder ausschließlich mittels künstlicher Besamung, die übrigen 14 (entsprechend 78 Proben) über Landbelegstellen. Dementsprechend erfolgte bei den Berechnungen eine Untergliederung in die Gruppen Hessen $(B)=$ Belegstelle und Hessen $(I)=$ instrumentelle Besamung. Zur Probennahme dienten nur solche Völker, die entweder nach erfolgter Körung als Reinzuchtvölker ausgewiesen waren oder seitens des Züchters zur Körung als Zuchtvölker vorgesehen waren.

Als Vergleichsmaterial für die vorliegende Untersuchung dienten die in der biometrischen Datenbank Oberursel abgelegten Daten von 23 Carnica-Proben aus dem alpinen Bereich und 15 Mellifera-Proben aus Norwegen. 


\section{Datenerhebung}

Für alle Hessenproben wurde zunächst die vollständige Merkmalsbestimmung für Arbeitsbienen entsprechend den Zuchtrichtlinien des Deutschen Imkerbundes (1988) in der Verfahrensweise nach Ruttner $(1983,1988 b)$ durchgeführt. Sie umfaßt die Merkmale Cubitalindex, Haarlänge auf der 5.Rückenschuppe, Filzbindenbreite auf der 4.Rückenschuppe und Panzerzeichen am Abdomen. Darüber hinaus wurden entsprechend dem Schema von Dupraw (1965a, b) diejenigen Flügelmerkmale bestimmt, welche für die Vergleichsproben aus der Datenbank Oberursel erfaßt waren. Es sind dies folgende Winkel: A4, B4, D7, E9, G18, K19, J10, J16, L13, N32, O26.

Die Messungen erfolgten auf der am Institut für Bienenkunde Oberursel für diesen Zweck eingerichteten Computeranlage (Meixner, 1994). Die zwischen zwei Objektträgern fixierten linken Vorderflügel wurden mit einer CCD-Kamera aufgenommen und auf einem Meßmonitor sichtbar gemacht. Mit Hilfe der Maus des Computers wurden in festgelegter Reihenfolge die benötigten Adernetzpunkte markiert, so daß das Programm die einzelnen Winkelmaße ableiten und speichern konnte. Von den einzelnen Proben wurden nur die Mittelwerte mittels Diskriminanzanalyse im Programmpaket SPSS-PC + V 4.01 analysiert. Informationen über die Verteilung der Einzelwerte wie zB Linksschiefe, Nebengipfel uä wurden in der Diskriminanzanalyse nicht berücksichtigt. Um jedoch einzelne Proben auf der Ebene der Einzelwerte mit den Datenbankwerten vergleichen zu können, wurden für die einzelnen Winkel sog. Winkelmaßklassen von je $1^{\circ}$ Klassenbreite gebildet, für welche dann Häufigkeitsverteilungen erstellt werden konnten. Das Verfahren entspricht sinngemäß dem in der Körpraxis üblichen Verfahren zur Beurteilung des Merkmals Cubitalindex einzelner Proben.

\section{ERGEBNISSE}

Die Untersuchung nach den Zuchtrichtlinien des Deutschen Imkerbundes (1988) ergibt für alle Proben den Befund 'rassetypisch'. Auffällig ist, daß die Werteverteilung für den Cubitalindex $(\mathrm{Cl})$ meist ausgeprägt oberhalb der Grenz- bzw Richtwerte der Zuchtrichtlinien liegt. (Danach sollen höchstens
$15 \%$ der Werte in die Klassen 15-17 und höchstens $2 \%$ in Klassen unter 15 fallen und der Mittelwert größer als 2,5 sein.) Die in Abbildung 1 wiedergegebene Zusammenfassung aller Werte in einer prozentualen Häufigkeitsverteilung zeigt sehr deutlich die Verschiebung gegenüber den in der Datenbank erfaßten Carnica-Werten. Die Untergruppen Hessen (B) und Hessen (I) unterscheiden sich in dieser Merkmalsverteilung nicht. Auf die nähere Darstellung der Befunde der anderen Körmerkmale wird hier verzichtet.

Nach Diskriminanzanalysen mit allen erfaßten Merkmalen zeigte sich ein sehr starker Einfluß des $\mathrm{Cl}$. In die weiteren Berechnungen wurde der $\mathrm{Cl}$ deshalb nicht mehr aufgenommen, weil er offensichtlich stark züchterisch beeinflußt ist (s. auch Bienefeld, 1992). Ebenso wurden die Winkel N23 und J16 aus der Berechnung genommen, weil ein Untersuchereffekt bei der Festlegung des gemeinsamen Meßpunktes zu vermuten war.

Abbildung 2 zeigt das Ergebnis der Diskriminanzanalyse mit den verbliebenen Merkmalen für die vier Gruppen $A$ m mellifera, A m carnica, Hessen (B) und Hessen (I). Die drei Carnica-Gruppen sind einander sehr ähnlich und alle deutlich von der $\mathrm{Mel}$ lifera-Gruppe abgesondert. Die beiden Hessengruppen setzen sich aber in der Achsrichtung von Funktion 2 tendenziell entgegengesetzt von der Carnica-Vergleichsgruppe ab. Tabelle I zeigt das Klassifikationsergebnis der Diskriminanzanalyse für die 4 Gruppen. Während die MelliferaProben eindeutig abgegrenzt werden, kommt es zwischen den einander ähnlichen Carnica-Gruppen zu Überlappungen. In Tabelle II sind für die 4 Gruppen die Mittelwerte für die einzelnen Merkmale aufgelistet.

Im Sinne des Vorgehens der Zuchtrichtlinien bei der Klassifizierung von Einzelproben wurde zunächst modellhaft für einige Proben und jeweils für die einzelnen Merk- 
male auch ein Vergleich auf der Ebene der Einzelwerte geführt. Als Beispiele werden hier die Einzelproben Nr 22 (Belegstelle) aus dem Zentrum der 'Hessenwolke' und $\mathrm{Nr} 111$ (Belegstelle) von dem der Mellifera zugewandten Rand der Hessenwolke ausgewählt. In den Abbildung $3 a, b$ sind für die beiden Proben die Häufigkeitsverteilungen je Winkelmaßklasse des Winkels A4 den jeweiligen Verteilungen der Gesamtheiten Carnica, Mellifera und Hessen gegenübergestellt. Probe 22 ist klar den einander sehr ähnlichen Verteilungen Carnica und Hessen zugeordnet, von denen sich Mellifera unterscheidet (Abb 3a). Probe 111 ist dagegen der Mellifera-Verteilung ähnlicher (Abb 3b).

Ähnliche Verhältnisse lassen sich auch für einzelne Proben der Datenbank-Carnica finden, welche in der Camica-Wolke im Randbereich zur Mellifera eingeordnet werden.

\section{DISKUSSION}

Nach den vorliegenden Diskriminanzanalysen werden die Proben aus den hessischen Reinzuchtbeständen sehr nahe bei der Carnica eingeordnet, nicht zwischen Carnica und Mellifera. Eine Hybridisierung zwischen Mellifera und Carnica ist hieraus nicht abzuleiten. Hinweise auf einen Hybridcharakter zwischen Carnica und Mellifera könnten sich allenfalls für einzelne Proben ergeben, wenn man sinngemäß wie beim Vorgehen der Zuchtrichtlinien für den Cubital-Index die Merkmalsverteilungen der Einzelproben mit der jeweiligen Verteilung der Gesamtheit - sozusagen als Standard vergleicht. Probe 111 wäre danach möglicherweise als Carnica-Mellifera-Hybride einzustufen. Diese Vorgehensweise bedarf aber noch der Absicherung durch Analysen gezielter Kreuzungsversuche. Vorläufig bleibt zu folgern, daß allenfalls sehr geringe Genanteile der Mellifera-Rasse in der hessischen Zuchtpopulation enthalten sind.
Wichtig für die weitere Betrachtung ist, daß die Hessenproben nicht deckungsgleich mit der typischen Carnica eingeordnet werden, und daß dabei die mittels instrumenteller Besamung geführten Bestände in eine andere Richtung tendieren als die über Belegstellen geführten Bestände. Für die Gruppe Hessen $(B)=$ Belegstelle ergibt sich im Verhältnis zu typischer Carnica und typischer Mellifera tendenziell eine ähnliche Einordnung, wie sie von Reinsch et al (1991) für die Niedersächsische Landbiene ermittelt wurde, allerdings aufgrund von anderen Merkmalen. Diese Autoren weisen darauf hin, daß der Genbestand der Landbiene nicht nur von Mellifera und Carnica, sondern auch von anderen Rassen bzw Herkünften (zB Caucasica, Buckfast) beeinflußt wird. Es kann somit vermutet werden, ist aber noch nicht durch Untersuchung der hessischen Landbiene belegt, daß von dieser Seite carnicafremde Gene in die Zuchtpopulation eingeflossen sind.

Für die Tendenz zur entgegengesetzten Einordnung der Gruppe Hessen $(I)=$ besamte ist eine Einkreuzung fremder Rassen als Ursache auszuschließen. Die 4 untersuchten Bestände zeigen diese Tendenz unterschiedlich stark. Der Bestand der Hessischen Landesanstalt für Tierzucht (Kirchhain) tendiert gegenüber der Vergleichsgruppe Carnica zu stärker negativen Werten der Funktion 1 (im Bild nach unten) und zu stärker positiven Werten der Funktion 2 (im Bild nach rechts). 2 aus diesem Bestand abgeleitete und dann unabhängig und relativ eng weitergeführte Gruppen bilden nahezu eigene 'Unterwölkchen' im Bereich hoher positiver Werte von Funktion 2 (siehe besondere Kennzeichnung in Abb 2). Diese Verteilung deutet eher auf zufällige Veränderungen im Sinne genetischer Drift.

Viele der über Belegstellen geführten Bestände aus Hessen haben im Lauf ihrer Entwicklung zeitweilig Reinzuchtmaterial aus Kirchhain zur Regeneration übernommen. Nähme man in der jetzigen Verteilung 
die (hier nicht ausgewiesene) Wolke Kirchhain statt der Wolke Carnica als Bezugsgröße, so erschiene für die Gruppe Belegstelle eine noch stärkere Abweichungstendenz in Richtung negativer Werte in Funktion 2 (im Bild nach links). Der hypothetisch angenommene Einfluß andersrassiger Genanteile (nicht an Carnica, nicht Mellifera) hätte damit noch ein stärkeres Gewicht. Effekte genetischer Drift sind allerdings auch nicht ganz auszuschließen.

In Bezug auf die Ausgangsfragestellung ist von Bedeutung, daß die Komponente Mellifera als ursprüngliches Hauptelement der Landbiene in der hessischen Zuchtpopulation Carnica nicht mehr in nennenswertem Umfang nachweisbar ist. Die Zuchtarbeit mittels Landbelegstellen und der zusätzlichen Merkmalskontrolle erscheint damit in Hessen, anders als in Niederbayern, als überwiegend erfolgreich. Wie weit die seit über 20 Jahren erfolgenden Vermehrungsleistungen aus besamten Beständen zu diesem positiven Ergebnis beigetragen haben, läßt sich nicht quantifizieren. Der Vergleich der über Belegstellen geführten mit den besamten Beständen legt aber die Annahme nahe, daß trotz einwandfreier Befunde gemäß den Zuchtrichtlinien bei den Belegstellen in gewissem Umfang fremde Genanteile in die Zuchtpopulation eingeflossen sind.

Die Unterschiede zum Ergebnis von Moritz (1991) erklären sich zT aus dem verschiedenen Untersuchungsmaterial: einerseits Stichproben aus niederbayerischen Reinzuchtgebieten, andererseits nach den Zuchtrichtlinien vorselektierte Proben aus hessischen Reinzuchtbeständen. ZT können die Unterschiede in den Ergebnissen aber auch durch die verschiedene Wahl der Referenzproben bedingt sein. Moritz (1991) hatte auf der Carnica-Seite 6 Proben aus dem Bestand der Hessischen Landesanstalt für Tierzucht (Kirchhain) eingesetzt mit der Begründung, der Bestand sei über künstliche Besamung geführt und schließe die meisten der zur Zeit in deutschen Carnica Zuchtprogrammen laufenden Linien ein. Das letztere trifft sicher nicht zu. Vor allem ist aber durch die vorliegende Untersuchung deutlich geworden, daß der Zuchtbestand Kirchhain sich stärker von Mellifera unterscheidet als die Vergleichsgruppe Carnica aus der Datenbank. Wäre der Cubitalindex in die Berechnung eingegangen, würde sich noch ein deutlicherer Unterschied zur Datenbank-Carnica zeigen. Auf der Mellifera-Seite stehen bei Moritz (1991) 7 Museumsproben, welche 1911/12 in Erlangen bzw Kulmbach konserviert worden waren. Er hat sie als Repräsentanten der damaligen Landbiene gewählt, gegen welche sich die seitherigen Zuchtbemühungen im Sinne einer Verdrängungszucht richteten. Ob es sich aber wirklich noch um ursprüngliche $A m$ mellifera ohne Einfluß von importiertem Material gehandelt hat, ist nicht sicher belegt. Deshalb wurde in der vorliegenden Untersuchung die Gruppe 'Norwegen' aus der Datenbank als Repräsentant für $A m$ mellifera gewählt. Nach Ruttner et al (1990) hat $A m$ mellifera während ihrer postglacialen Ausbreitung ein sehr einheitliches morphologisches Bild bewahrt, was ua auch durch den Vergleich mit Proben aus archaeologischen Funden belegt wird. Die Proben aus Erlangen konnten in die vorliegende Untersuchung nicht zu Vergleichszwecken einbezogen werden.

\section{SCHLUßFOLGERUNGEN}

Die Standardwerte der Zuchtrichtlinien sind vor allem auf die Abgrenzung gegen die Rasse Mellifera ausgerichtet. Sie orientieren sich heute noch an den von Ruttner (1969) im Ursprungsgebiet der Carnica erhobenen Werten. Um weiter im Sinne einer Überprüfung der Reinpaarung auf Landbelegstellen wirksam sein zu können, müßten sie sich aber eigentlich an den Ist-Werten der heutigen Zuchtpopulation orientieren, ins- 
besondere wegen des generell überhöhten Cubitalindex. Ersatzweise müßten andere Merkmale in die Überwachung einbezogen werden. Im Hinblick auf den dazu nötigen Aufwand muß sich die Züchterschaft fragen, ob dies sinnvoll sein kann, da eine absolut sichere und dauerhafte Kontrolle auf diesem Weg nicht möglich ist und mit Inselbelegstellen und Besamung gangbare Alternativen für den Kernbestand der Zuchtpopulation bestehen.

Diese Schlußfolgerung stimmt tendenziell mit der entsprechenden von Moritz (1991) überein, obwohl die eigentlichen Untersuchungsergebnisse sich beträchtlich unterscheiden.

\section{DANKSAGUNG}

Die Untersuchung erfolgte im Rahmen der Diplomarbeit von Andreas Hähnle unter Leitung von Prof Dr N Koeniger, Oberursel. Wir möchten Frau Dipl Biologin M Meixner für die Bereitstellung der Meßapparatur und der Zahlen aus der Datenbank Oberursel herzlich danken, ebenso Herrn Bienenzuchtberater N Petersen für die Unterstützung bei der Probennahme. Wir danken ferner Prof Dr N Koeniger, Oberursel und Dr R Büchler, Kirchhain, für die kritische Durchsicht des Manuskripts.

\section{Résumé - Étude morphométrique des ruchers de sélection en race pure d'Apis mellifera carnica en Hesse. En Allemagne} on utilise depuis longtemps la morphométrie pour contrôler les ruchers de sélection organisés à partir de la race $A m$ carnica. Le contrôle sert à reconnaître a posteriori d'éventuelles erreurs d'accouplement dans les stations de fécondation. Puisque seuls quelques caractères sont contrôlés, il se pourrait qu'à la longue des gènes de races étrangères s'introduisent sans qu'on s'en rende compte dans la population sélectionnée (Moritz, 1991). De ce point de vue, en Hesse où les accouplements se font en par- tie par insémination artificielle et en partie dans les stations de fécondation, il était nécessaire de tester la population sélectionnée. Dans un premier temps la pureté de la race, établie selon les directives du Deutscher Imkerbund (1988), a été testée sur un total de 123 échantillons de 30 abeilles chacun prélevés dans les populations de sélection en race pure de la Hesse. Par la suite on a déterminé pour la veination alaire les 10 angles suivants, qui sont des caractères non pris en compte dans la pratique apicole: A4, B4, D7, E9, G18, K19, J10, J16, L13, N32, O26. À titre de comparaison, on a pris dans la banque de données d'Oberursel les valeurs de 23 échantillons Carnica des Alpes et de 15 échantillons Mellifera de Norvège. Tous les échantillons étudiés étaient typiques de la race selon les directives de sélection fixées. L'index cubital comme caractère de contrôle présente une augmentation frappante influencée par la sélection par rapport aux valeurs d'origine (fig 1); ceci est dû à la surévaluation de ce caractère par les sélectionneurs. Dans l'image fournie par l'analyse discriminante des caractères non contrôlés par les sélectionneurs, les échantillons de la Hesse sont très proches des échantillons carnica d'origine (fig 2). On n'observe pas de tendance à se déplacer vers $A m$ mellifera mais il y a une tendance à dériver dans d'autres directions et, dans ces cas, les ruchers où est pratiquée l'insémination artificielle et les ruchers des stations de fécondation vont dans des directions opposées. Chez ces derniers les écarts par rapport à l'image caractéristique de Carnica sont dus principalement aux effets de la dérive génétique. Dans les ruchers des stations de fécondation les écarts au type carnica plaident plutôt pour l'existence de gènes étrangers à carnica, qui peuvent par exemple provenir de l'abeille Buckfast ou d'autres races importées. Contrairement à l'analyse discriminante, les valeurs individuelles des caractères permettent de mettre en évidence des ressemblances avec l'abeille autochtone 
A m mellifera. Le procédé n'est représenté ici à titre d'exemple que pour l'angle $\mathrm{A} 4$ sur 2 échantillons choisis. A $m$ carnica et $A m$ mellifera se différencient très bien par la répartition de la fréquence de l'angle A4 (figs $3 a$ et $3 b$ ). On compare les méthodes et les résultats avec ceux des études équivalentes faites par Moritz (1991) et Reinsch et al. Bien qu'on soit arrivé à un meilleur résultat de sélection en Hesse qu'en Bavière (Moritz, 1991), le présent travail montre lui aussi les limites de garantie du contrôle morphométrique des accouplements dans les stations de fécondation.

\section{A m carnica / A m mellifera / morphomé- trie / sélection en race pure / contrôle accouplement}

Table I. Classification results of discriminant analysis corresponding to figure 2 .

Tabelle I. Klassifikationsergebnis der in Abbildung 2 dargestellten Diskriminanzanalyse.

\begin{tabular}{|c|c|c|c|c|c|}
\hline \multirow[t]{2}{*}{ Group } & \multirow[t]{2}{*}{ Total } & \multicolumn{3}{|c|}{ Classified as } & \multirow[b]{2}{*}{ Hessen (I) } \\
\hline & & A m carnica & A m mellifera & Hessen (B) & \\
\hline A m carnica & 23 & $\begin{array}{l}19 \\
82.6 \%\end{array}$ & $\begin{array}{l}0 \\
0 \%\end{array}$ & $\begin{array}{l}2 \\
8.7 \%\end{array}$ & $\begin{array}{l}2 \\
8.7 \%\end{array}$ \\
\hline A $m$ mellifera & 15 & $\begin{array}{l}0 \\
0 \%\end{array}$ & $\begin{array}{l}15 \\
100.0 \%\end{array}$ & $\begin{array}{l}0 \\
0 \%\end{array}$ & $\begin{array}{l}0 \\
0 \%\end{array}$ \\
\hline Hessen (B) & 78 & $\begin{array}{l}9 \\
11.5 \%\end{array}$ & $\begin{array}{l}0 \\
0 \%\end{array}$ & $\begin{array}{l}54 \\
69.2 \%\end{array}$ & $\begin{array}{l}15 \\
19.2 \%\end{array}$ \\
\hline Hessen (I) & 45 & $\begin{array}{l}1 \\
2.2 \%\end{array}$ & $\begin{array}{l}0 \\
0 \%\end{array}$ & $\begin{array}{l}9 \\
20.0 \%\end{array}$ & $\begin{array}{l}35 \\
77.8 \%\end{array}$ \\
\hline
\end{tabular}

Total $76,4 \%$ correct classification.

Insgesamt $76,4 \%$ richtige Zuordnung.

Table II. Means and standard deviations of the tested characteristics.

Tabelle II. Mitte/werte und Standardabweichungen der untersuchten Merkmale.

\begin{tabular}{lrrrr}
\hline & & & \\
Characteristic & A m carnica & A m mellifera & Hessen $(B)$ & Hessen $(I)$ \\
\cline { 2 - 5 } & & & & \\
A4 & $29.0 \pm 2.0$ & $32.6 \pm 2.2$ & $29.2 \pm 3.7$ & $28.8 \pm 2.9$ \\
B4 & $112.7 \pm 5.5$ & $104.2 \pm 5.3$ & $110.6 \pm 5.9$ & $113.7 \pm 5.7$ \\
D7 & $99.3 \pm 3.0$ & $105.4 \pm 3.1$ & $97.3 \pm 4.1$ & $99.1 \pm 3.6$ \\
E9 & $23.3 \pm 1.5$ & $18.8 \pm 1.5$ & $23.1 \pm 2.3$ & $24.5 \pm 2.4$ \\
G18 & $92.7 \pm 2.5$ & $100.1 \pm 3.3$ & $90.4 \pm 3.9$ & $90.7 \pm 3.0$ \\
J10 & $52.5 \pm 3.1$ & $49.4 \pm 3.6$ & $53.5 \pm 3.6$ & $53.4 \pm 3.5$ \\
J16 & $96.4 \pm 3.4$ & $97.2 \pm 3.3$ & $91.9 \pm 3.6$ & $90.9 \pm 3.4$ \\
K19 & $79.4 \pm 2.8$ & $80.5 \pm 3.4$ & $78.2 \pm 4.3$ & $77.9 \pm 3.6$ \\
L13 & $12.3 \pm 1.2$ & $15.8 \pm 1.3$ & $13.1 \pm 2.1$ & $12.9 \pm 2.4$ \\
N23 & $94.4 \pm 2.8$ & $93.0 \pm 2.8$ & $91.7 \pm 4.3$ & $90.9 \pm 3.0$ \\
O26 & $37.9 \pm 3.6$ & $37.0 \pm 3.5$ & $36.5 \pm 4.6$ & $36.2 \pm 4.5$ \\
CJ & $2.6 \pm 0.3$ & $1.7 \pm 0.2$ & $3.2 \pm 0.6$ & $3.2 \pm 0.6$ \\
& & & & \\
\hline
\end{tabular}




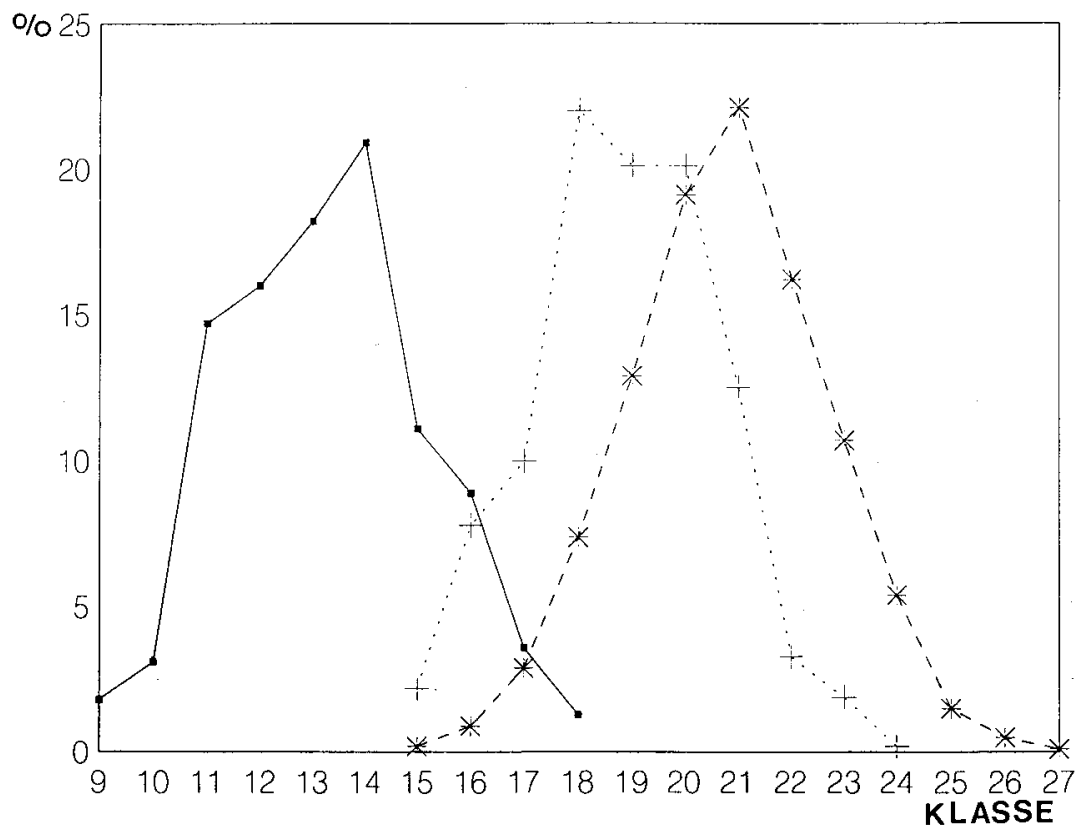

Fig 1. Relative frequency of cubital index according to Ruttner (1963) for $A m$ carnica $(\bullet+\bullet \cdot), A m$ mellifera (- - ) , and Carnica Hessen (- - -- ).

$\boldsymbol{A} \boldsymbol{b} \boldsymbol{b}$ 1. Relative Häufigkeit der Cubitalindexwerte in der Klasseneinteilung nach Ruttner für A $\mathrm{m}$ carnica $(\bullet+\bullet)$, A m mellifera (—), und Carnica Hessen (- -

FUNCTION 1

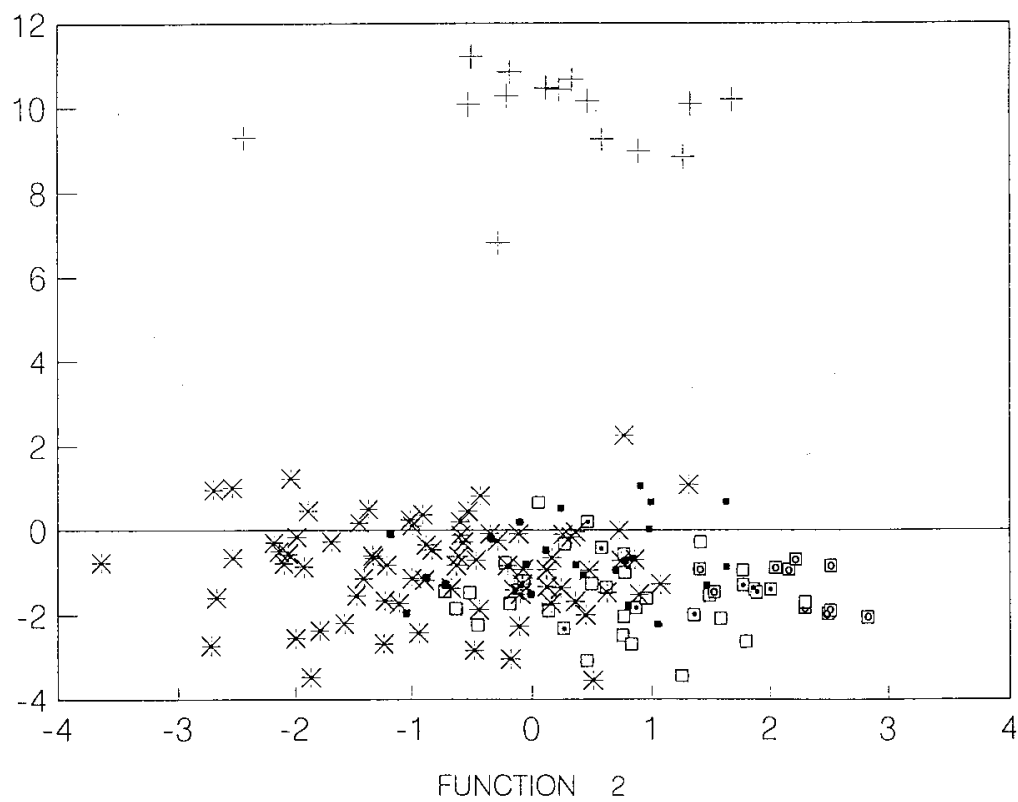

Fig 2. Discriminant analysis of the four groups: A m carnica (-); A m mellifera (+); Carnica Hessen (B) (*); and Carnica Hessen (I) ( $\square$ ). Marks in squares indicate two "subclusters" within the group Hessen $(I)=$ inseminated.

Abb 2. Diskriminanzanalyse der 4 Gruppen: A m carnica (-); A m mellifera (+); Carnica Hessen (B) (淑; und Carnica Hessen (I) ( $\square$ ). Markierte Quadrate Kennzeichnen zwei 'Unterwölkchen' innerhalb der Gruppe Hessen $(I)=$ besamte. 

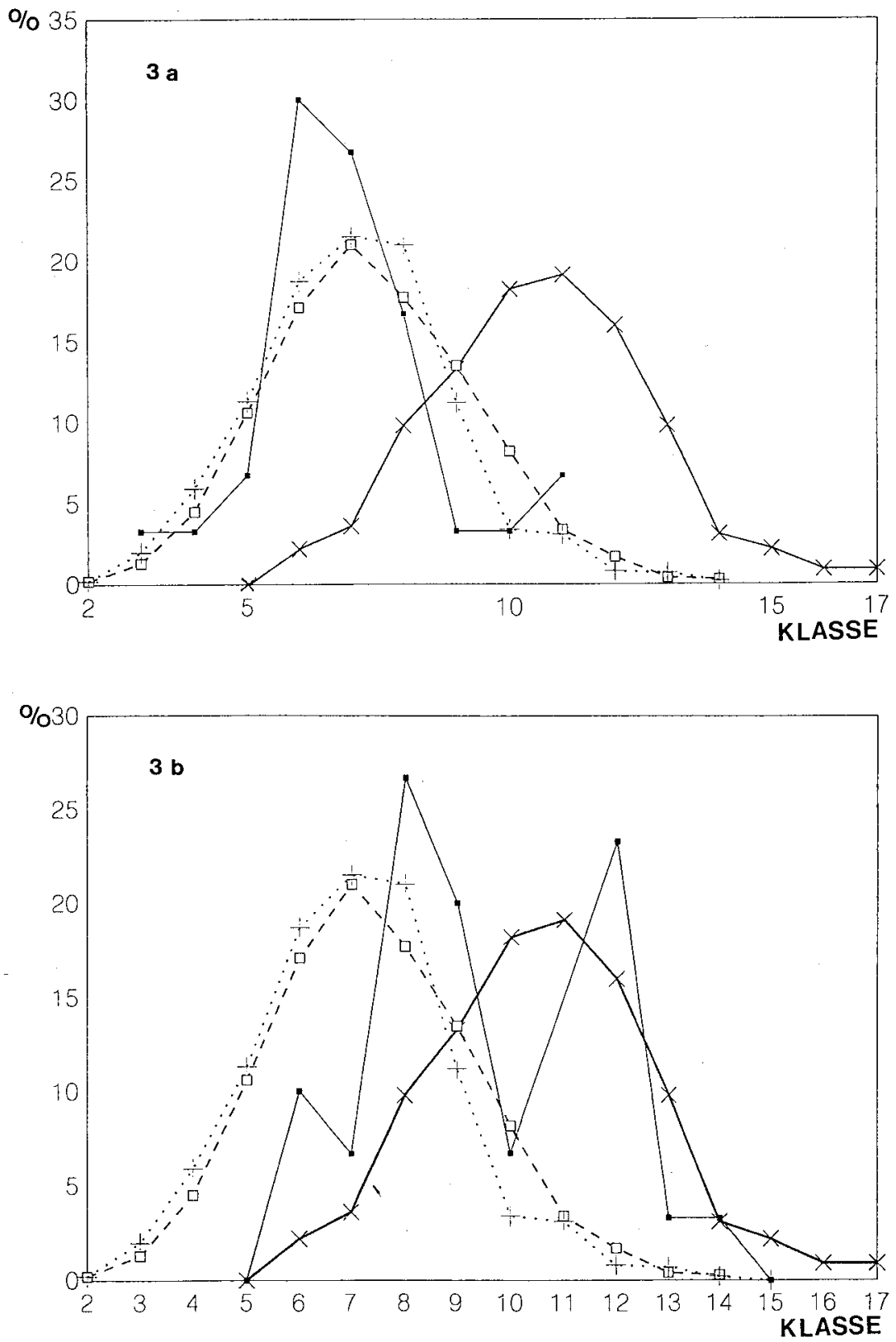

Fig 3. a,b. Relative frequency of values of angle A4 for: A m carnica (+...+); A m mellifera (-x-); Carnica Hessen (….. ). a. Single sample 22 from the center of the Hessen cluster ( - -); width of classes $1^{\circ}$. b. Single sample 111 from the border of the Hessen cluster toward Mellifera ( $\left.\square\right)$; width of classes $1^{\circ}$.

Abb 3. a,b. Relative Häufigkeit der Werte des Winkels A4 für: A m carnica $(+\ldots .+)$; A m mellifera

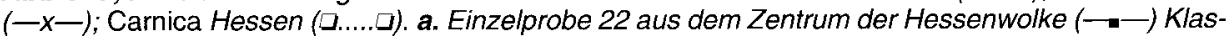
senbreite $1^{\circ}$. b. Einzelprobe 111 von Mellifera-seitigen Rand der Hessenwolke (一) Klassenbreite $1^{\circ}$. 


\section{REFERENCES}

Bienefeld K (1992) Der Cubitalindex: Genetische Grundlagen und züchterische Bedeutung. Bienenvater 113, 434-437

Deutscher Imkerbund (1954) Richtlinien für das Zuchtwesen des Deutschen Imkerbundes. Dtsch Bienenwirtschaft 5, 100-106

Deutscher Imkerbund (1971) Richtlinien für das Zuchtwesen des Deutschen Imkerbundes. Eigendruck

Deutscher Imkerbund (1988) Richtlinien für das Zuchtwesen des Deutschen Imkerbundes. Eigendruck

Dreher K (1946) Gedanken zum Neuaufbau des Zuchtwesens. Die Hessische Biene 81, 62$64,79-80$

Dreher K (1950) Beiträge zur Merkmalsuntersuchung der Honigbiene. $Z$ Bienenforsch 1, 17-23

Dupraw E (1965a) Non-Linnean taxonomy and the systematics of honeybees. Syst Zool 14, 124

Dupraw $E$ (1965b) The recognition and handling of honeybee specimens in non-Linnean taxonomy. J Apic Res 4 (2), 71-84

Goetze G (1940) Die beste Biene. Liedioff, Loth \& Michaelis, Leipzig

Meixner M (1994) Morphometrische, allozymatische und mt DNA Variabilität als Basis der innerartlichen Taxonomie am Beispiel von europäischen und afrikanischen Honigbienen (Apis mellifera L). Dissertation, JW Goethe, Universität, Frankfurt/Main

Moritz RFA (1991) The limitation of biometric control on pure race breeding in Apis mellifera. J Apic Res 30 (2), 54-59

Reichsfachgruppe Imker eV (1935) Der Aufbau der deutschen Königinnenzucht. Nr 1, Das Zuchtwesen. Verlag Rfgr Imker eV, Berlin

Reinsch N, Schuster H, Bienefeld K, Pirchner F (1991) Morphologischer Vergleich von Völkern der "Landbiene" in Niedersachsen mit typischer Apis mellifera carnica und Apis mellifera mellifera. Apidologie 22 (1), 75-80

Ruttner F (1963) Zuchttechnik und Zuchtauslese bei der Biene. Ehrenwirth Verlag, München

Ruttner F (1969) Biometrische Charakterisierung der Österreichischen Carnica-Biene. Z Bienenforsch 9, 469-491

Ruttner F (1983) Zuchttechnik und Zuchtauslese bei der Biene (5. Aufl) Ehrenwirth-Verlag, München

Ruttner F (1988a) Biogeography and Taxonomy of Honeybees. Springer-Verlag, Berlin

Ruttner F (1988b) Breeding Techniques and Selection for Breeding of the Honeybee. Codnor (British Isles Bee Breeders Association), UK

Ruttner F, Milner E, Dew JE (1990) The Dark European Honey Bee. Codnor (British Isles Bee Breeders Association), UK 